Publicación cuatrimestral. Vol. 5, No. 4 (Número Especial), Año 2021. Pág. 31-40

USO DEL INTERNET Y SU IMPACTO EN LA EDUCACIÓN DEL CANTÓN JIPIJAPA

\title{
USO DEL INTERNET Y SU IMPACTO EN LA EDUCACIÓN DEL CANTÓN JIPIJAPA
}

\author{
AUTORES: $\quad$ Darling Lenin Ponce Baque ${ }^{1}$ \\ Kirenia Maldonado Zúñiga ${ }^{2}$
}

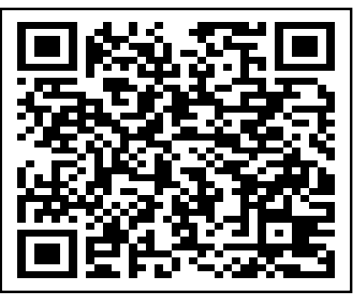

DIRECCIÓN PARA CORRESPONDENCIA: (ponce-darling6093@unesum.edu.ec)

Fecha de recepción: 9/03/2021

Fecha de aceptación: 22/05/2021

\section{RESUMEN}

Se realizó un estudio para determinar que el internet es un elementos tecnológicos utilizado en los actuales momentos para el desarrollo de las actividades que realiza el ser humano en la vida diaria, permitiendo realizar acciones que beneficien a los ciudadanos en diversos campos como el laboral, estudiantil, medicas, entre otros, con el uso de tecnologías y aplicaciones permitiendo la interacción con usuarios mediante videos conferencias y teletrabajo que son las modalidades que se desempeña en tiempos difíciles de restricciones y pandemia que se vive a nivel mundial. La educación virtual es uno de los temas que han prevalecido en los últimos días ya que permite formarse en distintos ámbitos que están ligados con la utilización del internet mediante el cual interactuamos con aplicaciones que faciliten la comprensión, análisis, interpretación de temas tratados para obtener conocimientos. Tiene como importancia el desarrollo de actividad en la educación porque mediante el internet y plataformas tecnológicas, los estudiantes han avanzado con sus actividades académicas y la utilización de recursos técnicos que permite afianzar sus habilidades y destrezas obteniendo resultados en su vida profesional. La educación es la preparación constante para la superación profesional y se enlaza con los valores éticos, morales y normas impartida en casa para formar a personas de bien. Se aplicó un diseño bibliográfico que permita identificar cual es la situación del uso del internet en el cantón Jipijapa así mismo indica su impacto en la formación académica.

PALABRAS CLAVE: académicas, conferencia, tecnologías, virtual.

\footnotetext{
${ }^{2}$ Máster en Ciencias de la Educación, Licenciada en Educación Informática. Docente de la carrera en Ingeniería en Tecnologías de la Información.. Universidad Estatal del Sur de Manabí. Jipijapa, Manabí, Ecuador. kirenia.maldonado@unesum.edu.ec, https://orcid.org/0000-0002-3764-5633
} 
Ponce Baque Darling Lenin, Kirenia Maldonado Zuñiga

\section{INTERNET USE AND ITS IMPACT ON EDUCATION IN THE JIPIJAPA CANTON}

\section{ABSTRACT}

A study was carried out to determine that the internet is a technological element used at the present time for the development of activities carried out by human beings in daily life, allowing actions that benefit citizens in various fields such as work, student, medical, among others, with the use of technologies and applications allowing interaction with users through video conferences and teleworking, which are the modalities that are carried out in difficult times of restrictions and pandemics that are experienced worldwide. Virtual education is one of the topics that have prevailed in recent days since it allows training in different areas that are linked to the use of the internet through which we interact with applications that facilitate the understanding, analysis, interpretation of topics covered to obtain knowledge. The development of activity in education is important because through the internet and technological platforms, students have advanced with their academic activities and the use of technical resources that allow them to strengthen their abilities and skills, obtaining results in their professional lives. Education is the constant preparation for professional improvement and is linked to ethical and moral values and standards taught at home to train good people. A bibliographic design was applied that allows identifying the situation of internet use in the Jipijapa canton, as well as indicating its impact on academic training.

KEYWORDS: academic, conference, technologies, virtual.

\section{INTRODUCCIÓN}

Las tecnologías en tiempos actuales es uno de los recursos más utilizados ya que permite interactuar en distintos campos de la informática, además, la interacción con otras personas y la utilización de plataformas, programas y sistemas nos ayudara a manejar criterios informáticos y de conocimientos en las labores diaria de los usuarios que son los que direccionan y manejan las búsquedas en las sistemas informáticos y web.

Internet permite a los usuarios de computadoras acceder remotamente a otros equipos y almacenes de información fácilmente, donde quiera que estén. Pueden hacer esto con o sin la seguridad informática. Está situado entre los implementos más utilizados en los actuales momentos en la sociedad, el país y el mundo ya que mediante esta herramienta se puede tener acceso a innumerable información que nos facilitara las actividades académicas y aplicarlas en algunos ambiente de la vida laboral como el teletrabajo, telemedicina, las clases en líneas entre otros, facilitando la interacción y cumplir con la educación que es un ámbito en donde se ve más reflejo el uso mediante aplicaciones tecnologías que permiten las interacción.

En los tiempos actuales y complicados por los que atraviesa el planeta con una declaratoria de emergencias a nivel del mundo han cambiado la manera de realiza las cosas y por el confinamiento en el que vivimos por la pandemia del covid 19, los seres humanos hemos tenido que adaptar a la nueva normalidad. En la búsqueda que las actividades no paralicen han examinado alternativas consientan que no perjudique y más bien ayuden a desarrollar acciones que permitan a los estudiantes recibir clases mediante plataformas didácticas y de interacción para compartir conocimientos y afianzar las enseñanzas impartidas por esta modalidad.

Internet, como la conocemos hoy en día, es una infraestructura de información muy difundida, el prototipo inicial de lo que se llama a menudo la Infraestructura de Información Nacional. Su historia es compleja e implica muchos aspectos: tecnológicos, organizativos y comunitarios. Y su

32 UNESUM-Ciencias. Publicación cuatrimestral. Vol. 5, Año 2021, No. 4 (Número Especial) 
influencia no solo alcanza los campos técnicos de las comunicaciones informáticas, sino también a toda la sociedad, ya que nos movemos hacia un uso mayor de las herramientas en línea para el comercio electrónico, la obtención de información y las operaciones comunitarias.

Además, la educación se define como un proceso a través del cual, los individuos adquieren conocimientos, ya sea habilidades, creencias, valores o hábitos, de parte de otros quienes son los responsables de transmitírselos, usando distintos métodos, por ejemplo, mediante discusiones, narraciones de historias, investigación y la formación. Desde un punto de vista técnico, el concepto de educación describe a un proceso continuo, en el que se desarrollan las facultades intelectuales, morales y físicas del hombre, con el objetivo de que se incorpore de manera eficiente en la sociedad o en el grupo donde se desenvuelve, por tanto, se puede decir que es un aprendizaje para la vida.

\section{DESARROLLO}

\section{HISTORIA DE INTERNET}

Antes de la creación de Internet, la única forma de comunicarse digitalmente era por medio del telégrafo El telégrafo se inventó en 1840, emitía señales eléctricas que viajaban por cables conectados entre un origen y un destino. Utilizaba el código Morse para interpretar la información.

\section{De ARPANET a WWW}

A principios de los 80 se comenzaron a desarrollar los ordenadores de forma exponencial. EL crecimiento era tan veloz que se temía que las redes se bloquearan debido al gran número de usuarios y de información transmitida, hecho causado por el fenómeno e-mail. La red siguió creciendo exponencialmente como muestra el gráfico.

WWW

El World Wide Web (WWW) es una red de "sitios" que pueden ser buscados y mostrados con un protocolo llamado HyperText Transfer Protocol (HTTP).

El concepto de WWW fue diseñado por Tim Berners-Lee y algunos científicos del CERN (Conseil Européen pour la Recherche Nucléaire) en Ginebra. Estos científicos estaban muy interesados en poder buscar y mostrar fácilmente documentación a través de Internet. Los científicos del CERN diseñaron un navegador/editor y le pusieron el nombre de World Wide Web. Este programa era gratuito. No es muy conocido actualmente, pero muchas comunidades científicas lo comenzaron a usar en su momento. (Barcelona, s.f.)

Cuál fue la idea original de la que surgió el internet

Internet fue el resultado de un experimento del Departamento de Defensa de Estados Unidos, en el año 1969, que se materializó en el desarrollo de ARPAnet, una red que enlazaba universidades y centros de alta tecnología con contratistas de dicho departamento. Tenía como fin el intercambio de datos entre científicos y militares. A la red se unieron nodos de Europa y del resto del mundo, formando lo que se conoce como la gran telaraña mundial (World Wide Web).

La idea y desarrollo de esta red se remonta a la creación de un proyecto en el que una red de computadoras permitiese la comunicación general entre los usuarios de distintas computadoras, tanto para el desarrollo de nuevas tecnologías, como para la fusión de la infraestructura de la red que ya existía, así como también los sistemas de telecomunicación. Los primeros datos 
relacionados con la interacción social que se realizó por medio del networking se encuentran contenidos en una serie de documentos que fueron editados por J.C.R Licklider, un informático estadounidense que trabajó para el Massachusetts Institute of Technology, en el año 1962, en dichos textos analiza y abre un debate acerca de su propio concepto, la red galáctica. (Adrián, 2019)

\section{INTERNET, ¿QUÉ ES? ¿PARA QUÉ SIRVE?}

Internet es una red que conecta a otras redes y dispositivos para compartir información.

Esto lo hace por medio de páginas, sitios o softwares. Su popularidad se ha hecho cada vez mayor por su capacidad de almacenar, en un mismo lugar, información de todo tipo y para diferente público. En Internet podemos encontrar información de música, arte, cultura, medicina, literatura, política, ingeniería y mucho más. Por medio de texto, audio, video, música, e imágenes, Internet nos permite informarnos, aprender y divertirnos.

Esta red es considerada por muchos como una herramienta perfecta para eliminar las jerarquías, las diferencias y las distancias, ya que favorece la libertad de expresión y permite que la información, el conocimiento y el mundo entero estén al alcance de un mayor número de personas*. Internet, entonces, no es una herramienta tecnológica más, es un medio de comunicación, interacción y organización social.

Internet es un espacio en el cual se reflejan los distintos ámbitos de la vida humana: la política, la educación, el esparcimiento, las cosas buenas y las cosas malas. Aquí, como en la vida real, el anonimato no existe, cualquier cosa que se envíe, se escriba o se suba en Internet, puede ser guardado y rastreado. Aquí, también como en la vida real, nos exponemos a peligros para los cuales se hace necesario tener unos comportamientos adecuados y responsables hacia lo que vemos, leemos y compartimos. La sinceridad, el respeto y la tolerancia son valores importantes a tener y promover cuando usamos la red. (enticconfio, 15)

Cosas para hacer en Internet:

- Investigar y aprender: bibliotecas, museos y libros gratuitos a través de la red.

- Comunicarnos y mantenernos en contacto con amigos y familiares que viven lejos usando chats, video-llamadas, correos electrónicos y redes sociales.

- Opinar libremente en blogs, páginas y redes sociales y saber lo que otros opinan.

- Compartir nuestros gustos e intereses con otras personas.

- Informarnos, en tiempo real, sobre lo que sucede en el país y el mundo.

- Realizar gestiones burocráticas y financieras desde la casa u oficina.

- Tomar cursos en instituciones y universidades virtuales.

- Escuchar música, ver videos y películas.

- Descargar libros, artículos, películas y música.

- Comprar en distintos almacenes, sin importar en qué lugar del mundo se encuentren. (enticconfio, 15)

Tipos de conexiones a Internet

$\mathrm{Al}$ ser Internet una red, se puede acceder a ella mediante varios canales.

Líneas telefónicas. Se puede utilizar una línea telefónica que se subdivide en líneas telefónicas convencionales (transmite señales de forma analógica pero actualmente fueron desplazadas por otras que ofrecen mayores beneficios) o digitales (mediante el empleo de un adaptador de red se traducen las tramas resultantes de la computadora a señales digitales).

34 UNESUM-Ciencias. Publicación cuatrimestral. Vol. 5, Año 2021, No. 4 (Número Especial) 
Publicación cuatrimestral. Vol. 5, No. 4 (Número Especial), Año 2021. Pág. 31-40 USO DEL INTERNET Y SU IMPACTO EN LA EDUCACIÓN DEL CANTÓN JIPIJAPA

Cable (fibra óptica). Asimismo, puede ser una conexión por cable (que implementa señales luminosas en vez de eléctricas que codifican una mayor cantidad de información y a su vez, dicho cable es de un material llamado fibra óptica que le permite transmitir datos entre nodos).

Satelital. También, existe la conexión vía satélite (normalmente estamos hablando de un sistema de conexión híbrido de satélite y teléfono para disminuir la congestión presente en las redes terrestres).

Redes inalámbricas. A su vez, podemos implementar redes inalámbricas (que sustituyen los cables por señales luminosas infrarrojas u ondas de radio para transmitir información). En suma, dentro de las redes inalámbricas, otro método utilizado es el de LMDS (que es una sigla que significa Local Multipoint Distribution System y consta de utilizar ondas de radio de alta frecuencia).

Líneas eléctricas. Si hablamos de líneas eléctricas usamos PLC (conjunción de tres palabras: Power Line Communications). Por último, podemos usar a la telefonía móvil (que refiere a comunicaciones disponibles para celulares para acceder a Internet). (Raffino, concepto.de, 2020)

\section{DEFINICIÓN DE EDUCACIÓN}

La educación puede definirse como el proceso de socialización de los individuos. Al educarse, una persona asimila y aprende conocimientos. La educación también implica una concienciación cultural y conductual, donde las nuevas generaciones adquieren los modos de ser de generaciones anteriores.

El proceso educativo se materializa en una serie de habilidades y valores, que producen cambios intelectuales, emocionales y sociales en el individuo. De acuerdo al grado de concienciación alcanzado, estos valores pueden durar toda la vida o sólo un cierto periodo de tiempo.

En el caso de los niños, la educación busca fomentar el proceso de estructuración del pensamiento y de las formas de expresión. Ayuda en el proceso madurativo sensorio-motor y estimula la integración y la convivencia grupal.

La educación formal o escolar, por su parte, consiste en la presentación sistemática de ideas, hechos y técnicas a los estudiantes. Una persona ejerce una influencia ordenada y voluntaria sobre otra, con la intención de formarle. Así, el sistema escolar es la forma en que una sociedad transmite y conserva su existencia colectiva entre las nuevas generaciones.

La educación hacia la que se tiende en la actualidad apunta a que cada alumno reciba un trato personalizado para que aprenda aquello que realmente le sirve y de la manera más adecuada a sus capacidades. Por ejemplo, a los futuros escritores no deberían exigirles un nivel de matemáticas propio de un científico. (Gardey, 2020.)

¿Qué es la educación?

Se denomina educación a la facilitación del aprendizaje o de la obtención de conocimientos, habilidades, valores y hábitos en un grupo humano determinado, por parte de otras personas más versadas en el asunto enseñado y empleando diversas técnicas de la pedagogía: la narración, el debate, la memorización o la investigación.

La educación es un proceso complejo en la vida del ser humano, que ocurre fundamentalmente en el seno de la familia y luego en las distintas etapas de la vida escolar o académica que el individuo transite (desde el kindergarten hasta la universidad). 
Sin embargo, no solo el conocimiento organizado y compartimentado de las ciencias y los saberes es educación: también lo son las tradiciones locales, las creencias familiares o los modos de conducta heredados.

$\mathrm{Y}$, sin embargo, el acceso a este tipo de educación suele estar restringido a las clases medias y altas, lo cual supone siempre una dificultad adicional para las clases más desfavorecidas, a menudo sumergidas en la ignorancia.

La educación puede darse a través de modelos distintos y diversos rangos de experiencias, pero generalmente está a cargo de un tutor, profesor, maestro o guía, que es una figura de relativa autoridad sobre los aprendices o estudiantes, encargada de velar por la correcta comprensión de los temas y de resolver las dudas que puedan surgir en el proceso, ya que no todas las personas tienen mecanismos de aprendizaje similares.

Conceptos de educación

Dependiendo del autor consultado, la educación se define como:

"La educación consiste en dirigir los sentimientos de placer y dolor hacia el orden ético" Aristóteles (filósofo griego, 384-322 a.C.)

"La consecución de un alma sana en un cuerpo sano, tal es el fin de la educación” John Locke (filósofo inglés, 1632-1704).

"La educación tiene por fin el desarrollo en el hombre de toda la perfección que su naturaleza lleva consigo” Immanuel Kant (filósofo alemán, 1724-1804)

"La educación es la única manera de aprender a vivir para otros por el hábito de hacer prevalecer la sociabilidad por sobre la personalidad” -Auguste Comte (filósofo francés, 1798-1857)

"Educación es evolución, racionalmente conducida, de las facultades específicas del hombre para su perfección y para la formación del carácter, preparándole para la vida individual y social, a fin de conseguir la mayor felicidad posible” - Rufino Blanco (educador español, 1861-1936)

"La educación es un proceso de transmisión de las tradiciones o de la cultura de un grupo, de una generación a otra” - Fernando de Azevedo (educador brasileño, 1894-1974) (Raffino, concepto.de, 2020)

Tipos de educación según el sector

De acuerdo con el sector que la administra, la educación puede ser pública o privada. En cualquier de estos dos casos, la educación deberá corresponder al proyecto de sociedad amparado en el marco legal del Estado como garante de la calidad y conveniencia social del proyecto educativo.

Educación pública

La educación pública es aquella impartida a través de las instituciones de Estado y suele tener un carácter formal. Por ser de interés público, el servicio educativo que imparte el Estado no tiene fines de lucro, sino que su propósito es estratégico.

En lo que refiere a la primaria y secundaria, el Estado debe proporcionar educación pública, gratuita y obligatoria. Respecto de la educación universitaria, según el país, el Estado puede ofrecer universidades gratuitas o, en su defecto, universidades que requieran una inversión significativamente menor que las privadas, a fin de favorecer la capacitación y promoción de los sectores populares al campo profesional.

Educación privada

La educación privada es aquella impartida en instituciones particulares. Puede abarcar tantas iniciativas en la enseñanza no formal y formal, así como diferentes niveles de esta última

36 UNESUM-Ciencias. Publicación cuatrimestral. Vol. 5, Año 2021, No. 4 (Número Especial) 
(educación básica, intermedia o superior). Estas instituciones poseen fines de lucro. (Imaginario, 2019.)

MATERIALES Y MÉTODOS

Para la elaboración del presente artículo los materiales utilizados para llevar a cabo el presente trabajo científico fueron diversas fuentes bibliográficas e investigación que se realizó para darle soporte bibliográfico basándose en criterios probados.

\section{MÉTODOS TEÓRICOS}

Estos métodos se utilizaron para el desarrollo de toda la investigación.

Histórico-lógico: se utilizó para la búsqueda de los antecedentes con énfasis en el ámbito del internet y descartar conceptos erróneos y datos erróneos.

Análisis - síntesis: se utilizó para determinar limitaciones, insuficiencias, falencias y potencialidades sobre el uso del internet, además, de la continua evolución que ha tenido para el funcionamiento de diversas plataformas.

\section{MÉTODOS EMPÍRICOS}

Revisión bibliográfica

Se utilizó en la fase de recopilación de información de libros, revistas de carácter científico, internet, los cuales contienen información del tema para la elaboración del presente artículo.

\section{RESULTADOS Y DISCUSIÓN}

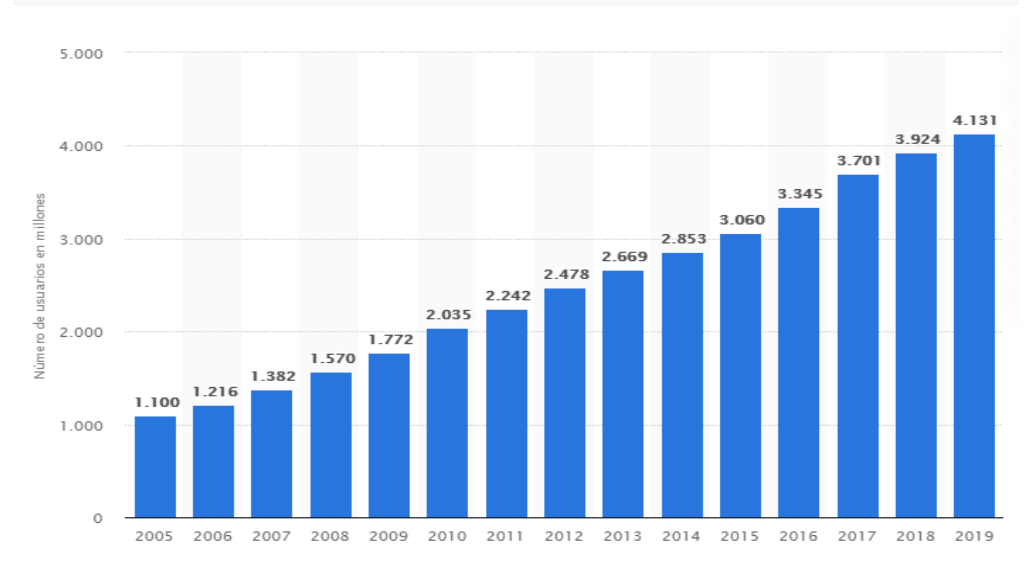

Figura 1

Fuente: Número de usuarios de Internet en el mundo entre 2005 hasta 2019 (Fernández, 2020)

Análisis: Esta estadística presenta la evolución anual del número de usuarios de Internet en el mundo desde 2005 hasta 2019. Durante ese periodo, el número de internautas creció paulatinamente hasta situarse por encima de los 4.100 millones en 2019. Este valor supone un aumento en más de 100 millones con respecto al año anterior. Por lo tanto los países desarrollados son los que tiene más acceso a las conexiones del internet. 


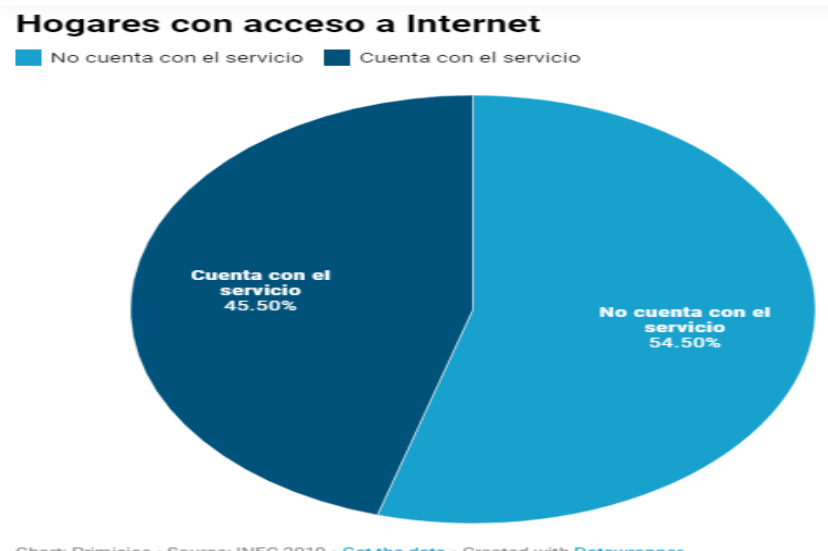

\section{Figura 2}

Fuente: Solo el 45,5\% de hogares en Ecuador tiene acceso a Internet, según el INEC (Dávalos, 2020)

Análisis: El Instituto Nacional de Estadísticas y Censos (INEC) presentó los datos sobre tecnologías de la información y comunicación 2019. Las cifras evidencian que en el país aún existe desigualdad tanto en el acceso a recursos tecnológicos como a servicios. Esto marca una brecha en la zona rural que, en la urbana, Ecuador ocupaba la mayor penetración de Internet en los países más poblados de Latinoamérica, liderando la lista.

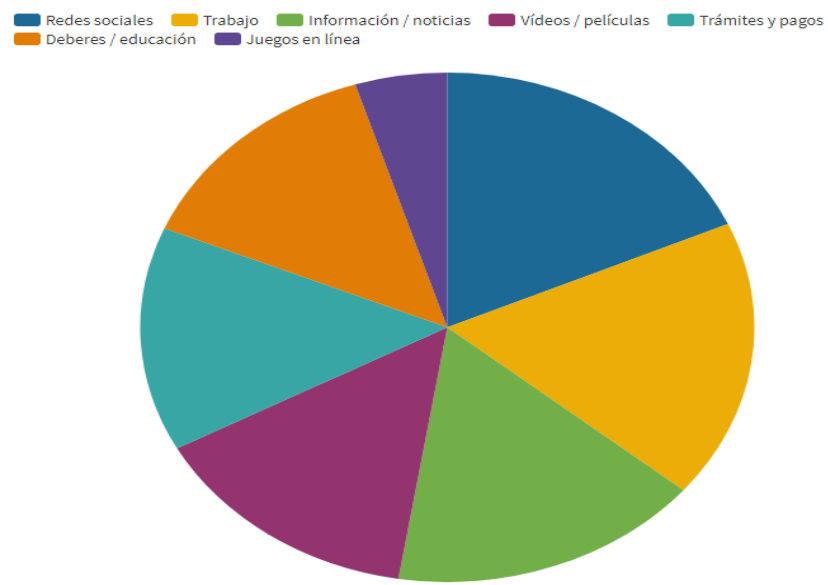

\section{Figura 3}

Fuente: Así es el uso de Internet en Ecuador (ESPINOZA, 2019)

Análisis: El Instituto Nacional de Estadísticas y Censos (INEC) presentó los datos sobre tecnologías de la información y comunicación 2019. Según el portal expreso en el Ecuador las personas más usan las redes sociales $82.5 \%$, trabajo $79.5 \%$, información/noticias $74.2 \%$, vídeos/películas 65,5 \%, trámites y pagos 64 \%, deberes/educación 62 \%, juegos en línea 21.8 \% y otros $1,3 \%$.

38 UNESUM-Ciencias. Publicación cuatrimestral. Vol. 5, Año 2021, No. 4 (Número Especial) 
En el análisis de lo ante propuesto nos indica que muchas personas a nivel mundial están contratando los servicios del internet para conectarse por los distintos medios electrónicos ya que en tiempos actuales estos está beneficiando en distintas modalidades de trabajo, mientras que en países en vías de desarrollo sus habitantes en las zonas rurales no tiene acceso a este servicio en su totalidad por zonas que son de difícil acceso o por la calidad de la señal en nuestro país como es el Ecuador según los estudios del (INEC) la población rural es uno de los más afectados ya sea por zonas de difícil acceso o por la calidad de vida de la población que no permite poder contratar porque el costo no lo pueden cubrir. Según los análisis y la búsqueda de información nos muestra en el Ecuador su habitante tienen más acceso a las redes sociales y realiza sus navegaciones en esta plataforma de entretenimiento, mientras que para el ámbito educativo tiene un porcentaje de $62 \%$ la cual nos indica que otras labores tiene más importancia al momento de realizar las búsquedas en el internet.

\section{CONCLUSIONES}

El uso de Internet se ha vuelto clave en los últimos tiempos, a propósito de las medidas que se han aplicado por la emergencia sanitaria causada por el covid-19. La cual permite interactuar de una manera diferente además de brindar un servicio vital en actividades relacionadas a teletrabajo, educación virtual, entretenimiento, trámites, compras y otros.

Hoy se tiene la posibilidad de enviar información de un lugar del mundo a otro en segundos, realizar presentaciones online, compartir la vida a través de fotos, vídeos, sonidos y textos, vivir en un mundo paralelo, pero, sobre todo, nuestra identidad auténtica. De esta manera, historias personales se convirtieron en públicas y temas locales se convierten en globales. Pero sobre todo nos muestra una diversidad de información que permite la adquisición de conocimientos y preparación intelectual para el futuro con miras a días mejores.

\section{REFERENCIAS BIBLIOGRÁFICAS}

Adrián, Y. (2 de septiembre de 2019). conceptodefinicion. Obtenido de conceptodefinicion: https://conceptodefinicion.de/internet/

Barcelona, F. d. (s.f.). retro-informatica. Obtenido de retro-informatica: https://www.fib.upc.edu/retroinformatica/historia/internet.html

Dávalos, N. ( 29 de Ago de 2020). primicias. Obtenido de https://www.primicias.ec/noticias/tecnologia/ecuadorhogares-acceso-internet-inec/

enticconfio. (12 de Sep de 15). enticconfio. Obtenido de https://www.enticconfio.gov.co/internet-que-es-para-quesirve

ESPINOZA, G. (29 de 11 de 2019). expreso. Obtenido de https://www.expreso.ec/ciencia-y-tecnologia/internetecuador-479.html

Fernández, R. ( 19 de oct. de 2020). statista. Obtenido de https://es.statista.com/estadisticas/541434/numeromundial-de-usuarios-de-internet/

Gardey, J. P. (2020.). definicion.de. Obtenido de https://definicion.de/educacion/

Imaginario, A. (2019 de 09 de 2019.). Significados.com. Obtenido de https://www.significados.com/educacion/

Raffino, M. E. (21 de octubre de 2020). concepto.de. Obtenido de https://concepto.de/internet/

Raffino, M. E. (8 de julio de 2020). concepto.de. Obtenido de https://concepto.de/educacion-4/ 
Ponce Baque Darling Lenin, Kirenia Maldonado Zuñiga

Verdezoto, J. M. (2021). derechoecuador.com. Obtenido de https://www.derechoecuador.com/delitos-informaticoso-ciberdelitosG

40 UNESUM-Ciencias. Publicación cuatrimestral. Vol. 5, Año 2021, No. 4 (Número Especial) 